\title{
Éditorial
}

\section{Dix enjeux pour l'agriculture en Méditerranée}

\section{Bertrand Hervieu}

Secrétaire général

Centre international

de hautes études agronomiques

méditerranéennes (Ciheam)

11, rue Newton,

75116 Paris

<hervieu@ciheam.org>
S i l'Europe souhaite pouvoir peser sur la scène internationale, elle ne peut ignorer le Bassin méditerranéen. L'interdépendance avec la Méditerranée est devenue telle que l'évidence de partenariats privilégiés s'impose. C'est par une coopération pionnière avec les autres pays méditerranéens que l'Europe sera en mesure de jouer un rôle dans la mondialisation, en explorant un avenir de codéveloppement durable où les variables humaines, sociales et environnementales seraient tout aussi déterminantes que les composantes économiques et politiques.

La multidimensionnalité de la question agricole et rurale en Méditerranée milite pour que soit enclenchée une mobilisation euroméditerranéenne sur ce sujet. Car l'agriculture se situe au cour de l'identité méditerranéenne et s'affiche comme un déterminant essentiel pour les économies et les sociétés de la région (Schmied, 2007).

Dix enjeux majeurs peuvent être identifiés pour dessiner les grands contours de l'agriculture méditerranéenne.

\section{Un contexte sociodémographique déterminant}

En l'espace d'un demi-siècle, la population méditerranéenne va presque doubler, passant de 285 millions d'habitants en 1970 à 525 millions à l'horizon 2020 Mais cette croissance démographique est cependant contrastée, car si le nord de la Méditerranée voit sa population se stabiliser depuis plusieurs décennies, à l'inverse, la rive sud connaît une explosion démographique impressionnante. En 2005, un tiers de la population en Méditerranée réside encore en milieu rural et un tiers des actifs dans les pays de la rive sud opèrent toujours dans le secteur agricole. Cette population rurale et agricole a naturellement gonflé sur la rive sud avec le boom démographique, tandis qu'au nord, parallèlement, la population rurale accélérait sa décroissance tout comme le nombre d'actifs agricoles. En ce début de XxI ${ }^{\mathrm{e}}$ siècle, ne nous trompons donc pas : la Méditerranée n'est pas uniquement urbaine, littoralisée, et axée sur les services.

\section{Une situation agrocommerciale préoccupante}

La dégradation des balances agrocommerciales des pays partenaires arabes méditerranéens mérite une attention toute particulière. En effet, depuis trois décennies, ces pays sont dans une situation chronique de dépendance alimentaire et certains États semblent désormais se situer dans des déficits structurels lourds (Algérie, Égypte). Ainsi, on constate un solde négatif de près de 9 milliards de dollars en 2004 pour les relations agrocommerciales des pays partenaires méditerranéens (PPM) ${ }^{1}$ avec l'ensemble du monde. Seule la Turquie présente en fait une balance positive, sachant qu'elle contribue pour $48 \%$ des exportations agricoles des PPM vers le monde.

\footnotetext{
${ }^{1}$ Dans le cadre du Processus de Barcelone les dix pays partenaires méditerranéens (PPM) de l'Union européenne sont : Algérie, Égypte, Israël, Jordanie, Liban, Maroc, Syrie, Tunisie, Turquie et Autorité palestinienne.
} 


\section{Un face à face euroméditerranéen en trompe-I'œil}

Occultée du débat euroméditerranéen depuis le lancement du Processus de Barcelone en 1995, la question agricole semble peu à peu se replacer dans l'agenda de la coopération régionale grâce aux initiatives prises ces derniers mois par la Commission et certains PPM. Mais si des négociations progressent pour la libéralisation des échanges avec une minorité d'États (Jordanie et Israël notamment, Égypte et Maroc ensuite), il faudrait clarifier les termes du débat agricole méditerranéen par trois messages importants.

D'abord, l'asymétrie des relations commerciales: l'Union européenne (UE25) commerce avec les dix PPM pour uniquement $2 \%$ de ses importations et exportations agricoles, mais polarise en revanche $52 \%$ de leurs exportations agricoles et couvre $28 \%$ de leurs importations. On a donc un différentiel très net entre le nord et le sud du Bassin en termes d'intensité agrocommerciale.

Ensuite, l'équilibre trompeur des échanges euroméditerranéens : ceux-ci sont favorables aux PPM (+ 0,6 milliard de dollars en 2004) simplement parce que la Turquie, à elle seule, contribue pour près de la moitié des exportations agricoles des PPM vers l'UE25. Résultat: sans la puissance agricole turque, la balance commerciale agricole des PPM est déficitaire avec l'Europe (1,5 milliard de dollars en 2004).

Enfin, l'ouverture des PPM sur le marché mondial: malgré leur préférence commerciale pour l'UE25, en 2004 ils se sont approvisionnés à $72 \%$ dans le reste du monde. L'Europe n'est donc pas l'unique puissance exportatrice vers le sud de la Méditerranée: États-Unis, Argentine, Brésil ou Australie sont des acteurs commerciaux importants, comme en attestent les exportations céréalières de ces pays vers les pays du sud de la Méditerranée. L'attitude du Maroc, qui signe en 2004 un accord de libre-échange avec Washington, montre par ailleurs que certains PPM cherchent aujourd'hui à nouer des alliances politico-commerciales hors du périmètre euroméditerranéen.

\section{Un mal-développement rural constaté}

L'urgence à développer les zones rurales du sud de la Méditerranée constitue un enjeu majeur dans la problématique régionale. Cet impératif reste prioritairement centré sur la lutte contre la pauvreté, fléau persistant dans les campagnes. Le nombre de personnes vivant avec moins d'un dollar par jour aurait même augmenté depuis 1990, décennie au cours de laquelle les effets des programmes d'ajustements structurels ont pesé sur le processus de développement de ces pays. Si au cours des décennies 1970 et 1980 des progrès importants avaient été enregistrés dans les pays méditerranéens du Sud, l'ennui, depuis les années 1990, provient non seulement du mal-développement qui se perçoit mais aussi du non-développement qui, parfois, se ressent (Ould Aoudia, 2006). Les infrastructures sociocollectives font défaut ou se dégradent (accès à l'eau, accès aux services sanitaires, accès à l'éducation), sans oublier l'inégalité des genres qui demeure toujours plus forte qu'en milieu urbain.

Dans ce milieu rural, il est difficile de repérer la construction d'une mise en marché efficace pour les produits agricoles: le manque d'organisation des producteurs, la faiblesse de la société civile ou les défaillances logistiques sont autant de freins au développement d'un système agroalimentaire performant. En réalité s'opposent toujours, au sud de la Méditerranée, une agriculture de firme d'un côté, déjà insérée dans la mondialisation et bien souvent contrôlée par des capitaux étrangers, et de l'autre, une agriculture familiale déstructurée, dont on peut craindre la lente décomposition si rien n'est proposé prochainement pour la remobiliser.

\section{Une fracture territoriale Sud-Sud qui s'accentue}

Dans la majorité des pays méditerranéens du Sud, le risque existe de voir à nouveau se déséquilibrer les territoires: c'est la réapparition d'une fracture intrarégionale. Dissimulé derrière les multiples failles qui traversent en longitude le Bassin méditerranéen, le clivage semble s'aggraver parallèlement entre les zones urbaines littorales et les espaces ruraux intérieurs.
À l'ouverture sur la mondialisation des villes répondent la marginalisation et la paupérisation des campagnes. Tout se passe comme si le sud de la Méditerranée n'avait plus besoin de ses arrière-pays pour se développer, le salut arrivant, selon un credo libéral discipliné, par les échanges et l'arrimage extérieur. Si les villes côtières sont propulsées sur l'avenir, à l'inverse, les territoires ruraux, quant à eux, sont renvoyés au siècle dernier.

\section{Un pluriel de défis environnementaux}

La richesse des ressources naturelles et la diversité des paysages de la Méditerranée en font une écorégion exceptionnelle de ce monde. Pourtant, avec le développement humain et industriel, ce patrimoine environnemental s'érode peu à peu. Malgré les efforts internationaux déployés depuis près de trente ans afin de protéger cet écosystème unique, celui-ci reste fragile et continue à se détériorer sous l'effet de l'augmentation des pressions exercées sur l'environnement.

L'impact du changement climatique, les menaces sur la biodiversité de la zone, l'érosion des sols ou les émissions polluantes provoquées par la consommation énergétique constituent des témoignages puissants de cette vulnérabilité croissante de l'espace méditerranéen. À cela s'ajoutent des phénomènes de sécheresse de plus en plus inquiétants et un processus de désertification aux conséquences dramatiques pour les territoires ruraux. Enfin, il convient de rappeler à quel point la zone méditerranéenne est confrontée aux incendies de forêt, dont les feux détruisent chaque année des pans entiers de surfaces agricoles.

\section{Une tension croissante sur les ressources en eau}

Au cœur des tensions écologiques, c'est la question de l'eau qui figure comme la plus préoccupante (Blanc, 2007). Cette ressource rare est très inégalement répartie en Méditerranée, avec $75 \%$ des disponibilités sur la rive nord. Aujourd'hui, la moitié de la population mondiale pauvre en eau (moins de $1000 \mathrm{~m}^{3} / \mathrm{hab} . / \mathrm{an}$ ) se trouve dans la région méditerranéenne. Alors que l'agriculture absorbe environ 
$80 \%$ des ressources en eau dans les pays de la rive sud et qu'une bonne partie de ce volume est perdue faute de réseaux d'adduction performants, une fracture hydraulique se renforce actuellement entre catégories aisées et pauvres pour qui l'accès à l'eau potable est sensiblement différent. Désormais, bien plus qu'en Europe, la qualité de l'eau est devenue dans ces pays un facteur de discrimination sociale.

Par ailleurs, il serait bon d'examiner en Méditerranée le coût écologique, et donc économique, des transferts d'eau contenus dans les produits agricoles (eau dite "virtuelle"), que certains pays, misant tout ou presque sur l'exportation, négligent sans doute trop à l'heure où les ressources s'épuisent. Enfin, comment ne pas craindre des rivalités plus tenaces entre États, régions ou communautés pour le contrôle des ressources en eau, sachant que dans certaines zones les conflits hydriques existent déjà ?

\section{Une sécurité alimentaire quantitative maîtrisée mais qualitativement fragilisée}

Face à l'ampleur de la croissance démographique dans la région et de la dégradation constatée des équilibres agrocommerciaux, la question de la sécurité alimentaire pour les populations méditerranéennes reste entière. Avec d'abord sa dimension quantitative, car la malnutrition reste un fléau non négligeable (environ $4 \%$ de la population du sud de la Méditerranée souffre d'une sousalimentation quotidienne) et numériquement en croissance ( 7 millions de personnes en 1990, 9 millions en 2002).

Ensuite, la dimension qualitative doit être réexaminée car l'alimentation méditerranéenne se dégrade, avec un alignement sur le modèle de consommation anglosaxon: le modèle crétois, vanté par l'Organisation mondiale de la santé (OMS), se délocalise et gagne les espaces occidentaux tandis que la Méditerranée s'en éloigne. À titre d'exemple, la forte progression de l'obésité et du surpoids, notamment chez les populations les plus jeunes (au Maghreb, $17 \%$ des enfants de moins de 5 ans). C'est dans ce contexte qu'il faut replacer la question de la sécurité sanitaire des aliments, et donc de la certification et des contrôles techniques, pour le thème désormais stratégique de la qualité alimentaire en Méditerranée (Centre international de hautes études agronomiques méditerranéennes, 2007).

\section{Une émergence rapide de la grande distribution}

Depuis une quinzaine d'années, la grande distribution s'affirme dans les pays du sud de la Méditerranée. La situation au Maghreb est éloquente (Abis et Padilla, 2007), puisque l'on est passé d'un paysage dépourvu en grandes centrales d'achat à un panorama urbain se recomposant autour de surfaces commerciales sans cesse plus attractives. L'essor d'une classe moyenne urbaine branchée sur les standards de consommation modernes et la hausse relative des niveaux de vie pour ces populations expliquent en partie ce phénomène.

Assurément, cette poussée de la grande distribution, même si elle ne représente encore que $10 \%$ du total des ventes alimentaires au Maghreb, risque de bousculer les pratiques industrielles de ceux qui ont décidé de collaborer, avec notamment l'écueil d'une industrie locale à deux vitesses. En effet, s'appuyant sur une industrie normalisée, une logistique organisée, des approvisionnements importants en volume et réguliers, la grande distribution alimentaire exige organisation et ressources à la fois humaines et financières. Autant d'efforts auxquels bon nombre d'industriels ne sont sans doute pas prêts à consentir.

\section{Un débat sur les biocarburants à clarifier}

La promotion des biocarburants et leur développement sont des faits mondiaux qu'il convient de reconnaître, avec les avantages que cela apporte parfois. Mais si l'on raisonne à l'échelle du Bassin méditerranéen, il serait vivement conseillé de ne pas trop s'enthousiasmer sur ce dossier. D'abord, l'agriculture dans la région, peut-être plus que partout ailleurs, ne pourra pas à la fois nourrir les hommes et remplir les réservoirs des voitures : la croissance démographique est trop importante, les surfaces agricoles manquent et les conditions naturelles sont inadaptées et tendanciellement défavorables. Ensuite, comment ne pas craindre que la Méditerranée soit l'illustration parfaite des faits récemment observés, à savoir une corrélation intime entre croissance des prix agricoles et développement des biocarburants?

Car pour produire de l'éthanol ou du biogazole, on utilise en effet de plus en plus des céréales, du sucre, des graines oléagineuses ou des huiles végétales, avec pour effet une augmentation notable des prix sur le marché. Or ce sont des produits massivement importés par les pays méditerranéens du sud de la Méditerranée : rappelons que ces États attirent $12 \%$ des importations mondiales de céréales alors qu'ils ne comptent que pour $4 \%$ de la population du globe. Ainsi, en se positionnant sur les biocarburants, les États-Unis, le Brésil ou l'Union européenne non seulement cherchent à transformer le marché des hydrocarbures, mais font augmenter le prix des céréales, ce qui les remet dans une position de complète domination vis-à-vis des pays du Sud.

\section{Conclusion}

Ce panorama non exhaustif des dynamiques agricoles, rurales et alimentaires en Méditerranée montre assez bien l'éventail des enjeux auquel l'agriculture est confrontée dans cette région. Il importe d'en considérer toutes les dimensions, du territorial au socioculturel en passant par la santé publique et la géopolitique, pour en comprendre le caractère éminemment stratégique.

Si le soin est pris de ne pas cloisonner le débat agricole méditerranéen dans les simples périmètres du commercial et de l'agronomique, peut-être pourrait-on sortir des malentendus qui pèsent sur cette question, et ainsi, au contraire, y explorer toutes les variables déterminantes pour le futur de la Méditerranée?

Une fois cette posture adoptée, peut-être pourrait-on construire et progresser dans la construction d'une coopération méditerranéenne fondée sur un triptyque santé mobilisateur : santé des économies (savoir optimiser et rationaliser les systèmes agricoles de chaque pays), santé des territoires (savoir protéger et préserver l'environnement), et santé des hommes (savoir se nourrir). 


\section{Références}

Schmied W. La politique agricole et rurale euroméditerranéenne. Strasbourg: Conseil de l'Europe, Commission de l'environnement, de l'agriculture et des questions territoriales, 2007.

Ould Aoudia J. Croissance et réformes dans les pays arabes méditerranéens. Notes et documents $n^{\circ} 28$. Paris: Agence française de développement (AFD), 2006.
Blanc P. Tensions méditerranéennes sur les ressources en eau. Lettre de veille CIHEAM $2007 ;(1): 1-4$

Centre international de hautes études agronomiques méditerranéennes (Ciheam). Mediterra 2007, Identité et qualité des produits alimentaires méditerranéens. Rapport annuel du Ciheam. Paris: Les Presses de Sciences-Po 2007
Abis S, Padilla M. La grande distribution au Maghreb : contextualisation et enjeux. AfkarIdeas (Madrid) 2007 ; (13) : 70-3.

United Nations Organisation for Food and Agriculture (FAO), Organisation for Economic Cooperation and Development (OECD). OECD FAO Agricultural Outlook 2007-2016. Rome : FAO, 2007.

\section{Centre international de hautes études agronomiques méditerranéennes (Ciheam)}

Le Centre international de hautes études agronomiques méditerranéennes (Ciheam) a été créé, à l'initiative conjointe de l'Organisation de coopération et de développement économiques (OCDE) et du Conseil de l'Europe, le 21 mai 1962. C'est une organisation intergouvernementale qui réunit aujourd'hui treize États membres du Bassin méditerranéen (Albanie, Algérie, Égypte, Espagne, France, Grèce, Italie, Liban, Malte, Maroc, Portugal, Tunisie et Turquie).

Le Ciheam se structure autour d'un secrétariat général situé à Paris (France) et de quatre Instituts agronomiques méditerranéens (Bari en Italie, Chania en Grèce, Montpellier en France, et Saragosse en Espagne).

Avec au cœur de son activité trois missions fondamentales (formation, recherche, coopération), le Ciheam s'impose désormais comme une référence dans son domaine d'activité : I'agriculture, l'alimentation et le développement rural en Méditerranée.

\section{Secrétariat général du Ciheam}

11, rue Newton - 75116 Paris (France)

+33 (0)153239100

www.ciheam.org

\section{Observatoire méditerranéen du Ciheam}

www.medobs.org 